\title{
THE GEOMETRY OF THE WEDDLE MANIFOLD $W_{p}{ }^{*}$
}

\author{
BY A. B. COBLE
}

During the last few years our secretaries have been so active in organizing interesting programs at the regular meetings that surveys of progress in the more important fields of mathematics have been presented to the Society quite recently. At the meeting last April in New York, reports on Algebraic Geometry were given. I have therefore felt impelled to change in some measure the traditional character of this biennial address, and to select a topic somewhat more special than usual. Lest this justification may seem too hollow, let me add that this change is in the direction of my personal interest in mathematics. For the benefit of those members present who are not specialists in geometry, I hope to illustrate by relatively simple early cases the general aspect of the problem, emphasizing those methods whose extension presents no difficulty. A technical account of the novelties presented will appear in the April number of the American Journal of Mathematics.

The Weddle manifold $W_{p}$ has the dimension $p$ in the linear space $S_{2 p-1}$, the first case for $p=2$ being the long known Weddle quartic surface in $S_{3}$. Let us consider first its group-theoretic character. We all are familiar with the quadratic Cremona involution,

$I_{y z}: \quad x_{i} x_{i}^{\prime}=y_{i} z_{i}, \quad\left(i=0,1,2 ; x, x^{\prime}\right.$ in the same plane $)$,

which has $F$-points at the vertices of the triangle of reference and which interchanges the points $y$ and $z$. Its fundamental importance is due to the fact that the entire group of Cremona transformations in the plane is generated by the group of collineations and by the single element $I_{y z}$ above. For every larger limit for $i,(i=0,1,2, \cdots, r)$, this involution $I_{y z}$ persists; and it, together with the group of collineations, generates the regular Cremona group in $S_{r}$. When $r \geqq 3$, this regular Cremona group is no longer the entire Cremona group of the space. The regular

* An address delivered at Pittsburgh, December 28, 1934, as the retiring presidential address, before the American Mathematical Society. 
Cremona transformations are characterized by the property that they are completely defined to within collineations at most by a finite number of $F$-points, the existence of other $F$-loci, $F$-curves, $F$-surfaces, etc., being a necessary consequence of the existence of these $F$-points.

Returning to $S_{2}$ we take $2+3$ points, $p_{1}, p_{2}, \cdots, p_{5}=P_{5}{ }^{2}$, and denote by $I_{12}$ that involution $I_{y z}$ which interchanges $p_{1}$ and $p_{2}$ and which has $F$-points at $p_{3}, p_{4}, p_{5}$. Then the ten involutions $I_{i j},(i, j=1, \cdots, 5 ; i \neq j)$, generate a finite abelian group of order $2^{4}$ and type $(1,1,1,1)$ whose elements multiply according to the following rules:

$$
I_{i j}{ }^{2}=1, I_{i j} I_{i k}=I_{j k}, I_{i j} I_{k l}=I_{k l} I_{i j}=I_{i k} I_{j l}=I_{i j k l} \text {. }
$$

It consists of the identity, the ten quadratic involutions, and five cubic involutions of type $I_{i j k l}$ each with one double and five simple $F$-points. Similarly in $S_{3}$, with $3+3$ points, $p_{1}, \cdots, p_{6}=P_{6}^{3}$, let $I_{12}$ be that cubic involution $I_{y z}$ which interchanges $p_{1}$ and $p_{2}$ and which has $F$-points at $p_{3}, \cdots, p_{6}$. We again obtain a finite abelian group of order $2^{5}$ with a multiplication table as above. Proceeding in this fashion we define in terms of $r+3$ points in $S_{r}$ an abelian group of order $2^{r+2}$.

There is however an interesting difference between the spaces of odd and of even dimension. When $r$ is odd, say $r=2 p-1$, the number of points being $2 p+2$, the abelian group contains a symmetric element $I_{12 \ldots 2 p+2}$ for which the $F$-points all have the same character. These symmetric regular Cremona transformations are very scarce. In every $S_{r},(r \geqq 2)$, there is the generating element $I_{y z}$. In every odd $S_{2 p-1}$ there is the element $I_{12 \ldots 2 p+2}$ just mentioned. Apart from these fairly generic types there are only three further symmetric types in $S_{2}$, one further type in $S_{3}$, and one in $S_{4}$. We define the procession of Weddle manifolds $W_{p},(p \geqq 2)$, to be the loci of fixed points of the involutions $I_{12} \ldots 2 p+2$. Thus the first Weddle $W_{2}$ in $S_{3}$ is the locus of fixed points of that space involution whose pairs are the seventh and eighth base points of nets of quadrics contained in the web on $P_{6}{ }^{3}$, these two points coming together on the locus of nodes of quadrics on $P_{6}{ }^{3}$.

Because of the abelian character of the finite Cremona $G_{2}{ }^{2 p+1}, W_{p}$ is invariant under the group, and on $W_{p}$ each point is invariant under the identity and $I_{1} \ldots 2 p+2$. Thus $G_{2}{ }^{2 p+1}$ effects 
upon $W_{p}$ the transformations of a $g_{2}{ }^{2 p}$ which is isomorphic with the additive group of half periods of the theta functions of genus $p$, so that some relation of $W_{p}$ to these functions may be expected. Since also the set of points $P_{2 p+2}^{2 p-1}$, which defines $I_{12} \ldots 2 p+2$ and therefore $W_{p}$, is on a unique rational norm-curve $N^{2 p-1}$, and may be projectively determined on this curve by a binary $(2 p+2)-\mathrm{ic},(\omega t)^{2 p+2}=0$, it is clear that the theta functions involved would necessarily be the hyperelliptic thetas.

Let us turn then to the hyperelliptic curves for a second definition of $W_{p}$. The normal form commonly used for the discussion of the integrals on such a curve is

$$
z^{2}=(\omega t)^{2 p+2}=\left(t t_{1}\right) \cdots\left(t t_{2 p+2}\right),
$$

the unique $g_{1}^{2}$ on the curve being given by $z,-z$. But another normal form has also been much used, namely, that for which the unique $g_{1}^{2}$ is cut out by a pencil of lines, that is

$$
H_{p}^{p+2} \equiv f_{p}\left(t_{1}, t_{2}\right) \cdot y_{0}^{2}+2 f_{p+1}\left(t_{1}, t_{2}\right) y_{0}+f_{p+2}\left(t_{1}, t_{2}\right)=0,
$$

$f_{p}, f_{p+1}, f_{p+2}$ being binary forms in $t_{1}, t_{2}$, of the orders indicated. This curve of genus $p$ and order $p+2$ has a $p$-fold point at $O\left(y_{0}: t_{1}: t_{2}=1: 0: 0\right)$. The branch lines of the $g_{1}{ }^{2}$ are given by

$$
(\omega t)^{2 p+2}=f_{p+1}^{2}-f_{p} f_{p+2}=\prod\left(t t_{i}\right)=0,
$$

and the $2 p+2$ branch points on the curve by

$$
y_{0}: t_{1}: t_{2}=-f_{p+1}\left(t_{i}\right): t_{i 1} f_{p}\left(t_{i}\right): t_{i 2} f_{p}\left(t_{i}\right) .
$$

The $3 p+5$ constants in the forms $f$ are reduced to $p+3$ if the branch lines, $(\omega t)^{2 p+2}=0$, are given. Three more constants can be removed by a projectivity which leaves fixed the $p$-fold point $O$ and the branch lines on it. Under these conditions $H_{p}{ }^{p+2}$ has $p$ absolute projective constants. The $\infty^{p}$ curves obtained by variation of these absolute projective constants are all birationally equivalent. It may be shown that every member of the aggregate $\left(\infty^{p}\right)$ may be obtained from a given one by a Cremona transformation and that such member is uniquely determined when a given $p$-ad of points on the given curve is required to pass into the $p$-ad at $O$. If the branch points of $H_{p}{ }^{p+2}$ are $r_{1}, \cdots, r_{2 p+2}$, then the set of $2 p+3$ points in $S_{2}$ consisting of these $2 p+2$ branch points and $O$ are associated with a set of 
$2 p+3$ points in $S_{2 p-1}$ of which $2 p+2$ are the set $P_{2 p+2}^{2 p-1}$ which defines $W_{p}$ and the last is a point $x$ on $W_{p}$ which runs over $W_{p}$ as the $p$-ad on the given $H_{p}^{p+2}$ varies.

I should like to comment briefly on this matter of the association of two sets of $n$ points. In one of the simplest problems of algebra, namely, the solution of a system of linear homogeneous equations in $n$ variables, we naturally drop the dependent equations, and we seek the solutions of the system of $r$ linearly independent remaining equations. These depend upon the matrix, $M_{r, n}=\left(a_{i j}\right)$, of coefficients of the system whose rank is $r$, say $r<n$ to avoid trivialities. The solutions of this system are given in terms of $n-r$ linearly independent solutions which may be arrayed in a matrix $M_{n-r, n}=\left(b_{i j}\right)$. Obviously these two matrices are reciprocally related, the row product of any row of the one with any row of the other is zero. Thus the determination of the solutions $\left(b_{i j}\right)$ of a system with coefficients $\left(a_{i j}\right)$ carries with it the determination of the solutions $\left(a_{i j}\right)$ of a system with coefficients $\left(b_{i j}\right)$. This interesting reciprocity between the two matrices, which I shall call apolar matrices, passes practically unnoticed in treatises on algebra despite its important applications in geometry. One of these applications, given by Grassmann in 1862, is to the determination of a linear space on the one hand by means of the points which it contains, and on the other by means of the linear spaces which contain it. Another application is found in the extensive theory of apolarity of linear systems of algebraic forms and of linear systems of algebraic loci. In analysis we find that the matrix $M_{n-1, n}$, whose rows are 0 th, 1 st, $\cdots,(n-2)$ th derivatives of the $n$ solutions of a linear differential equation of the $n$th order, has, for its apolar matrix $M_{1, n}, n$ solutions of the adjoint equation.

In the definition of apolar matrices the emphasis is on the rows. But, in a situation like this, a reflective geometer will not overlook the role played by the columns. The columns of $M_{r, n}$ may be interpreted as $n$ points in $S_{r-1}$, those of $M_{n-r, n}$ as $n$ points in $S_{n-r-1}$. These two sets of points, ordered with respect to each other, I have called associated sets of $n$ points. Since in either matrix the rows can be replaced by independent linear combinations of all the rows, each of the two associated sets is determined by the other set only to within projectivities. The proportionality of complementary determinants of orders 
$r, n-r$ in two apolar matrices shows that, if one set of points satisfies some geometric condition, the associated set must also satisfy some geometric condition, though this second condition is usually of a totally different geometric character from the first.

This association of two sets of $n$ points has a deeper significance than its projective definition would indicate. The association is in fact invariant under properly applied regular Cremona transformation. If an involution $I_{y z}$ with $F$-points at $r$ of the first set transforms the remaining $n-r$ points into new points, these $r F$-points and $n-r$ new points form a new set of $n$ points in $S_{r-1}$ congruent to the original set. In the associated set in $S_{n-r-1}$ take the $n-r$ complementary points as $F$-points of $I_{y z}$ to form a similar congruent set. Then the two new sets, congruent respectively to the original associated sets, are still associated. Thus, in the two associated sets previously mentioned, first of $P_{2 p+2}^{2 p-1}$ and $x$ on $W_{p}$ in $S_{2 p-1}$, and second of $r_{1}, \cdots, r_{2 p+2}$ and $O$ in $S_{2}$, the involution $I_{12}$ is associated with the perspective involution $A_{12}$ with $F$-points at $O, r_{1}, r_{2}$ and with directions at $r_{1}, r_{2}$ corresponding respectively to points on the lines $O r_{1}, O r_{2}$. To the involution $I_{12} \cdots 2 p+2$, of which $x$ is a fixed point, there is associated the product $A_{12} A_{34} \cdots A_{2 p+1,2 p+2}=J_{12 \cdots 2 p+2}$, and this is the de Jonquiéres involution for which $H_{p}^{p+2}$ is a locus of fixed points and for which the set of points $r_{i}, O$ is congruent to itself.

We use the association, proved in the above manner, to obtain the coordinates of the point $x$ on $W_{p}$. As a coordinate system in $S_{2 p-1}$ we take the $2 p$ coefficients of a binary $(2 p-1)$-ic, $(\alpha t)^{2 n-1}$, the perfect powers of linear forms then representing the points of a rational norm-curve $N^{2 p-1}$, which for present purposes we identify with the $N^{2 p-1}$ on the $2 p+2$ points $P_{2 p+2}^{2 p-1}$. Coordinate systems of this character date back to Weyr and his contemporaries. They are especially effective for all problems in which a rational norm-curve enters in a significant way, since then the highly developed algebra of binary forms in one or more sets of variables can be utilized.

It is sufficient by way of illustration to take the case $p=2$. The $H_{2}^{4}=f_{2} y_{0}^{2}+2 f_{3} y_{0}+f_{4}=0$ with double point $O$ has for matrix of coordinates of its six branch points and of $O$ : 


$$
\left(\begin{array}{rrrrr}
-f_{3}\left(t_{1}\right) & -f_{3}\left(t_{2}\right) & \cdots & -f_{3}\left(t_{6}\right) & 1 \\
t_{1} f_{2}\left(t_{1}\right) & t_{2} f_{2}\left(t_{2}\right) & \ldots & t_{6} f_{2}\left(t_{6}\right) & 0 \\
f_{2}\left(t_{1}\right) & f_{2}\left(t_{2}\right) & \ldots & f_{2}\left(t_{6}\right) & 0
\end{array}\right),
$$

$t$ here being non-homogeneous for convenience. The matrix of coordinates of the six points, $P_{6}{ }^{3}$, in $S_{3}$ on the norm-curve $N^{3}$ and of the point $x$ on $W_{2}$ is the matrix of coefficients of the seven binary cubics

$$
\left(t t_{1}\right)^{3},\left(t t_{2}\right)^{3}, \cdots,\left(t t_{6}\right)^{3},(\alpha t)^{3} .
$$

We wish to determine $(\alpha t)^{3}$ and such constant factors for the perfect cubes that these two matrices may be apolar. Let the linear factors of $f_{2}(t)$ be

$$
f_{2}(t)=\left(t r_{1}\right) \cdot\left(t r_{2}\right) .
$$

Then the linear identity connecting eight sixth powers reads:

$$
\begin{aligned}
\frac{\left(t t_{1}\right)^{6}}{f_{2}\left(t_{1}\right) \cdot \omega^{\prime}\left(t_{1}\right)}+\cdots & +\frac{\left(t t_{6}\right)^{6}}{f_{2}\left(t_{6}\right) \cdot \omega^{\prime}\left(t_{6}\right)}+\frac{\left(t r_{1}\right)^{6}}{\omega\left(r_{1}\right) \cdot f_{2}^{\prime}\left(r_{1}\right)} \\
& +\frac{\left(t r_{2}\right)^{6}}{\omega\left(r_{2}\right) \cdot f_{2}^{\prime}\left(r_{2}\right)} \equiv 0
\end{aligned}
$$

where $f_{j}^{\prime}(s)$ is the product of the differences of a particular root $s$ of $f_{j}$ and of each of the other roots of $f_{j}$. The polar of $f(t)$ as to this identity cancels the last two terms and removes the factor $1 / f_{2}\left(t_{i}\right)$ from the others yielding the identity connecting six fourth powers. This identity shows that the six perfect cubes of the second matrix must have factors $1 / f_{2}\left(t_{1}\right), \cdots, 1 / f_{2}\left(t_{6}\right)$, respectively, in order that the second matrix may be apolar to the last two rows of the first matrix. That it may be apolar to the first row also requires that

$$
(\alpha t)^{3}=\frac{f_{3}\left(t_{1}\right) \cdot\left(t t_{1}\right)^{3}}{f_{2}\left(t_{1}\right) \cdot \omega^{\prime}\left(t_{1}\right)}+\cdots+\frac{f_{3}\left(t_{6}\right) \cdot\left(t t_{6}\right)^{3}}{f_{2}\left(t_{6}\right) \cdot \omega^{\prime}\left(t_{6}\right)} .
$$

We have here then the coordinates of a point on the Weddle $W_{2}$ in $S_{3}$ in terms of the roots $t_{1}, \cdots, t_{6}$ of $(\omega t)^{6}=f_{3}^{2}-f_{2} f_{4}$. It is not the best form of $(\alpha t)^{3}$, since there are many linear relations connecting six cubes, and since $f_{3}$ and $f_{2}$ satisfying

$$
z_{t}^{2}=(\omega t)^{6}=f_{3}^{2}-f_{2} f_{4}
$$


cannot be chosen arbitrarily when $(\omega t)^{6}$ is given. But, for a root $r_{i}$ of $f_{2}, z_{r_{i}}=f_{3}\left(r_{i}\right)$. If then we polarize the above identity as to $f_{3}(t)$, the first six terms yield $(\alpha t)^{3}$, whence

$$
-(\alpha t)^{3}=\frac{\left(t r_{1}\right)^{3}}{z_{r_{1}} \cdot f_{2}^{\prime}\left(r_{1}\right)}+\frac{\left(t r_{2}\right)^{3}}{z_{r_{2}} \cdot f_{2}^{\prime}\left(r_{2}\right)} .
$$

As immediate generalizations we find that

$$
\begin{aligned}
(\alpha t)^{2 p-1} & =\sum_{i=1}^{i=2 p+2} \frac{\left(t t_{i}\right)^{2 p-1} \cdot f_{p+1}\left(t_{i}\right)}{f_{p}\left(t_{i}\right) \cdot \omega^{\prime}\left(t_{i}\right)}, \\
-(\alpha t)^{2 p-1} & =\sum_{j=1}^{j=p} \frac{\left(t r_{j}\right)^{2 p-1}}{z_{r_{j}} \cdot f_{p}^{\prime}\left(r_{j}\right)} .
\end{aligned}
$$

Since the coefficients $\alpha$ in this last formula are symmetric in the $p$ pairs of values $r_{i}, z_{r_{i}}$, the coordinates $x$ of a point on $W_{p}$ are proportional to abelian functions of $u_{1}, \cdots, u_{p}$ on $H_{p}{ }^{p+2}$ determined by the $p$-ad of points on $H_{p}{ }^{p+2}$ :

$$
r_{1}, z_{r_{1}} ; r_{2}, z_{r_{2}} ; \cdots ; r_{p}, z_{r_{p}} .
$$

The superposed p-ad for which all the $z$ 's change sign yields the same point $(\alpha t)^{2 p-1}$, whence the abelian functions are even.

The last formula expresses the coordinates of a point $x$ on $W_{p}$ in terms of the coordinates of the $p$ crossings of $N^{2 p-1}$ and of the unique $p$-secant $S_{p-1}$ of $N^{2 p-1}$ which passes through $x$. This $S_{p-1}$ is determined by $f_{p}=0$. The $S_{p-1}$ contains, however, a set of $2^{p-1}$ points $x$ of $W_{p}$ which arise from the variation of signs of $z_{r_{i}}$. We have then theorems of the following type:

The Weddle quartic $W_{2}{ }^{4}$ in $S_{3}$ contains $N^{3}$ on $P_{6}{ }^{3}$, and a bisecant of $N^{3}$ meets $W_{2}{ }^{4}$ in two further points harmonic to the two crossings of the bisecant.

The Weddle $W_{3}$ in $S_{5}$ of order 19 contains $N^{5}$ on $P_{8}{ }^{5}$ as a triple curve. Of the 19 points in which it meets a trisecant plane of $N^{5}$, 9 are found at the three triple points on $N^{5}$, and 6 at the contacts with $W_{3}$ of the three bisecants at their crossings. The remaining 4 are generic points of $W_{3}$ whose diagonal triangle is the three crossings of the trisecant plane.

Naturally this last theorem is in much more precise form than can be verified here. 
Each element of a finite Cremona group in $S_{2}$ has a certain number of $F$-points and corresponding $P$-curves. The aggregate of such $F$-points and $P$-curves for all elements of the group must divide into one or more sets such that in a set all the members are conjugate under the group. In space $S_{3}$ we shall have $F$-points, $F$-curves, and $P$-surfaces similarly disposed under a finite group. We lump all of these under the generic term $F$-loci of the group. For regular groups in $S_{3}$, however, the $F$-points and $P$-surfaces must lie in a different conjugate system from the $F$-curves. Thus, for the $G_{2.2}{ }^{4}$ of the Weddle $W_{2}$ defined by $P_{6}{ }^{3}$ the directions at the point $p_{1}$, say $\pi_{1}$, pass by $I_{56}$ into $\pi_{156}$, the plane on $p_{2}, p_{3}, p_{4}$; and this plane passes by $I_{34}$ into $\pi_{123456}$, the quadric with node at $p_{2}$ and on the other five points. These 6 points, 20 planes, and 6 nodal quadrics make up a conjugate set of $2 \cdot 2^{4} F$-loci of the first kind conjugate under $G_{2.2^{4}}$. Similarly the line $p_{1} p_{2}$, say $\pi_{12}{ }^{(2)}=\pi_{3456}{ }^{(2)}$, passes by $I_{12}$ into $\pi^{(2)}=\pi_{123456}{ }^{(2)}$, the cubic curve $N^{3}$ on $P_{6}{ }^{3}$. Again these 15 lines and one cubic curve make up a conjugate set of $2^{4} F$-loci of the second kind conjugate under $G_{2 \cdot 2^{4}}$. These $F$-loci of the second kind are on $W_{2}$ and each is invariant under $I_{12} \ldots 6$ which defines $W_{2}$. On the other hand the $2 \cdot 2^{4}$ loci of the first kind are paired by $I_{12 \ldots 6}, \pi_{1}$ with $\pi_{23456}$ and $\pi_{123}$ with $\pi_{456}$, and the intersection of each of the $2^{4}$ pairs is a locus on $W_{2}$. The product of the members of a pair is a quadric on $P_{6}{ }^{3}$. Quadrics on $P_{6}{ }^{3}$ map $W_{2}$ into the Kummer quartic surface, the $F$-loci of the first kind of $W_{2}$ contributing the 16 tropes of the Kummer surface, and the $F$-loci of the second kind contributing the 16 nodes of the Kummer surface. Each locus of one kind is on $2 p+2=6$ loci of the other kind.

Similarly, $W_{3}$ has three kinds of $F$-loci each forming a conjugate set of $2 \cdot 2^{6}$, except for $2^{6}$ of the third kind which are on $W_{3}$. Those of the first kind have dimension 0 or 4 ; of the second kind, dimension 1 or 3 ; of the third kind, dimension 2 . The mapping system which converts $W_{3}$ into the generalized hyperelliptic Kummer 3-way of Klein and Wirtinger is a system of cubic spreads with nodes at $P_{8}{ }^{5}$, the $F$-spaces of the first kind of dimension 0 , and simple points on the $F$-spaces of the second kind of dimension 1. Thus it turns out that the hyperelliptic Kummer 3-way in $S_{7}$ has, in addition to 64 singular points and 64 singular $S_{6}$ 's, also a set of 64 singular $S_{3}$ 's. 
Generically, then, $W_{p}$ has $p$ kinds of pairs of $F$-loci with the following incidence relations: Each pair of $F$-loci of the $j$ th kind, $(j=1, \cdots, p)$, contains $2 p+2$ pairs of $F$-loci of the $(j+1)$ th kind and is contained by $2 p+2$ pairs of $F$-loci of the $(j-1)$ th kind. Thus the hyperelliptic Kummer $p$-way $K_{p}$ has, in addition to the $2^{2 p}$ singular points and $2^{2 p}$ singular spaces given by the classic theta function theory, $p-1$ sets of $2^{2 p}$ singular linear spaces of intermediate dimension with the incidence relations just mentioned.

The mapping system which converts $W_{p}$ into $K_{p}$, a system of spreads of order $p$ with $(p-1)$-fold points at $P_{2 p+2}^{2 p-1}$, has essential importance. If its dimension is less than $2^{p}-1$, then $W_{p}$ is mapped not into $K_{p}$ but rather into some projection of $K_{p}$ (I have shown elsewhere that the theta squares are represented by members of this system). When $p=2$, quadrics in $S_{3}$ on $P_{6}{ }^{3}$ have the dimension $9-6=2^{2}-1$; when $p=3$, cubics in $S_{5}$ with nodes at $P_{8}{ }^{5}$ have the dimension $C_{8,5}-8 C_{6,5}-1$ $=2^{3}-1$; when $p=4$ the argument is not so simple. Quartics in $S_{7}$ have the dimension $C_{11,7}-1=330-1$; the 10 triple points contribute $10 C_{9,7}=360$ conditions. However, if $p$ and $q$ are triple points of the quartic $(\alpha x)^{4}=0$, then $(\alpha p)^{2}(\alpha x)^{2} \equiv 0$ and $(\alpha q)^{2}(\alpha x)^{2} \equiv 0$. Thus the condition $(\alpha p)^{2}(\alpha q)^{2}=0$ is counted twice. The revised dimension is now $330-1-360+45=2^{4}-2$, and is not the expected $2^{4}-1$. The method of counting conditions just used is quite all right for 8 triple points, since $C_{11,7}-1-8.36+C_{8,2}=C_{8,4}-1$ and the system has the equation $\sum a_{i j k l} x_{i} x_{j} x_{k} x_{l}=0,(i, j, k, l=0, \cdots, 7)$. If one imposes on this system the further conditions that $y$ and $z$ be triple points (28 conditions for each with one dependence for the pair) there is certainly no easy way to see algebraically that the apparently independent 55 conditions are still dependent. Naturally such complications would increase with increasing $p$. If, however, the coordinate system with reference to $N^{2 p-1}$ on $P_{2 p+2}^{2 p-1}$ be employed, the members of the mapping system are represented by symmetric forms of order $2 p-1$ in $p$ sets of binary variables. One may then show with relative ease that the linear system $\Sigma$ of order $p$ with $(p-1)$-fold points at $P_{2 p+2}^{2 p-1}$ has the dimension $2^{p}-1$ and that it contains all the $F$-loci of the $j$ th kind and dimension $j-1$ to the multiplicity $p-j,(j=1, \cdots, p)$. Furthermore, the dimension of the sub-system of $\Sigma$ which con- 
tains a pair of $F$-loci (paired under $I_{12 \ldots 2 p+2}$ ) is also readily obtained so that the dimensions of the intermediate singular linear spaces of $K_{p}$ are likewise known.

Let us pass to a second series of loci on hyperelliptic $K_{p}$ which is suggested by $W_{p}$. There is a familiar planar theorem to the effect that if two generically placed rational envelopes of class $m$ and $n$ are put into generic one-to-one correspondence, the locus of the intersection of corresponding lines is a rational curve of order $m+n$. We say that the two envelopes generate the rational curve. The curve is then perspective to either envelope, that is, like named point of the one and line of the other are incident. This leads to a rough classification of rational curves of given order. If, for example, a rational septimic has a perspective cubic (necessarily unique), it is generated by this cubic and a perspective quartic. The septimic may, however, be specialized to the point of having a perspective conic. It then has no proper perspective cubic and must be generated by the perspective conic and a perspective quintic. The septimic may even have a perspective point, necessarily a six-fold point. It is then generated by this point and a perspective sextic. Thus the septimic must be one of three types, say type $(3,4)$, type $(2,5)$, or type $(1,6)$.

There is a somewhat analogous classification of hyperelliptic curves $H_{p}{ }^{p+2}$. If two such curves $H_{q}{ }^{q+2}, H_{r}{ }^{r+2}$ have a common center $O$, a line through $O$ meets them in two pairs of points with a common harmonic pair. The locus of this pair as the line turns about $O$ is a hyperelliptic $H_{q+r+1}^{q+r+3}$ on the branch points of $H_{q}{ }^{q+2}, H_{r}{ }^{r+2}$, with center $O$, and with branch points at the common points of these two curves. We shall say again that $H_{q+r+1}^{q+r+3}$ is generated by $H_{q}{ }^{q+2}, H_{r}^{r+2}$. The condition that $H_{q}{ }^{q+2}$ should serve as a generator of $H_{p}{ }^{p+2}$ is that the two have the same center $O$ and that either be on the branch points of the other. Analytically the condition is

$$
f_{p} g_{q+2}-2 f_{p+1} g_{q+1}+f_{p+2} g_{q} \equiv 0 .
$$

This imposes $q+p+3$ conditions of which $3 q+5$ can be absorbed by the three forms $g$, leaving $p-2 q-2$ conditions on the three forms $f$. Thus it is $p-2$ conditions that the branch points of $H_{p}{ }^{p+2}$ be on a conic, $p-4$ conditions that they be on a cubic through $O, p-6$ that they be on a quartic with node at $O$, etc. 
Hence there is on $W_{p}$ a locus $V_{2}(p)$ whose points represent $H_{p}{ }^{p+2}$ 's with branch points on a conic, a locus $V_{4}(p)$ whose points represent $H_{p}{ }^{p+2}$, s with branch points on a cubic, and thus a chain of loci $V_{2}(p), V_{4}(p), V_{6}(p), \cdots$ is defined on $W_{p}$.

The first of these, $V_{2}(p)$, has a number of the outstanding properties of the original Weddle $W_{2}$ with which it coincides when $p=2$. I give one example. By proper choice of the line of reference $y_{0}$ opposite $O$, the conic on the branch points of $H_{p}{ }^{p+2}$ can be given the form, $y_{0}^{2}-(a t)^{2}=0$. The above identity is now $f_{p} \cdot(a t)^{2}-f_{p+2}=0$, whence the fundamental $(2 p+2)$-ic is $(\omega t)^{2 p+2}=f_{p+1}^{2}-f_{p}^{2} \cdot(a t)^{2}=0$. Thus, for the branch parameters $t_{i}$, we have $f_{p+1}\left(t_{i}\right) / f_{p}\left(t_{i}\right)=\sqrt{\left(a t_{i}\right)^{2}}$, and the formula (A) given earlier becomes now

$$
(\alpha t)^{2 p-1}=\sum_{i=1}^{i=2 p+2} \frac{\sqrt{\left(a t_{i}\right)^{2}} \cdot\left(t t_{i}\right)^{2 p-1}}{\omega^{\prime}\left(t_{1}\right)}
$$

This for variation of the quadratic $(a t)^{2}$ yields the $\infty^{2}$ points of $V_{2}(p)$. It may be shown that the point given remains on $V_{2}(p)$ for arbitrary choice of signs of the $2 p+2$ radicals. If the sign of the first radical be changed a new point of $V_{2}(p)$ is obtained which is on a line with the original point and the singular point $p_{1}$. Hence we have the following theorem.

The line on a singular point of $V_{2}(p)$ and a point of $V_{2}(p)$ cuts $V_{2}(p)$ again; if then a point $x$ of $V_{2}(p)$ is projected from the $2 p+2$ singular points of $V_{2}(p)$ into $2 p+2$ new points of $V_{2}(p)$, if these new points are similarly projected into further points, etc., a system of $2^{2 p+1}$ points on $V_{2}(p)$ is obtained which is closed under such projection.

For $p=2$ and $V_{2}(p)=W_{2}$ this theorem has been given by H. F. Baker.

There are on $P_{2 p+2}^{2 p-1} \infty^{4}$ normal elliptic curves $E^{2 p}$. The mapping system $\Sigma$ cuts such an $E^{2 p}$ in a $g_{1}{ }^{2}$ which has four branch points on $W_{p}$. If $E^{2 p}$ is binodal and therefore degenerate, the locus of the nodes is $V_{2}(p)$; if it is not so degenerate, the locus of the $\infty^{4}$ branch points is $V_{4}(p)$. This exhausts the possibilities connected with the family of elliptic curves, and I have not thus far found a generalization of this family which will serve for the isolation of $V_{6}(p), V_{8}(p)$, etc. 
In conclusion I wish to call attention to a third system of loci on $W_{p}$ which arises from extensions and generalizations of a theorem due to two of my associates of other days at Johns Hopkins, Professors Morley and Conner. There is a fairly old theorem relating to the Kummer surface $K_{2}$, with many interesting contacts, which states that the section of $K_{2}$ by a generic plane is a generic quartic curve. This might be expected since $K_{2}$ has three absolute constants and the plane adds three more. However, $W_{2}$, the Weddle quartic surface, also has three absolute constants, but its generic section, $Q$, as Morley and Conner pointed out twenty-five years ago (American Journal of Mathematics, vol. 31 (1909), p. 263), is not a generic quartic curve. They determine the invariant of degree six which $Q$ satisfies. They explain its geometric character by using a pencil of Weddle's as follows. The $W_{2}$ is localized by its hexad of nodes $P_{6}{ }^{3}$ on $N^{3}$, that is, by a binary sextic, $(\omega t)^{6}$. The pencil of binary sextics, $\lambda(\omega t)^{6}+\left[(\mu t)^{3}\right]^{2}$, determines a pencil of $W_{2}$ 's, and in particular the member determined by $\left[(\mu t)^{3}\right]^{2}$ contains the plane $(\mu t)^{3}$, trisecant to $N^{3}$, as a part. Thus the quartic section $Q$ of $W_{2}$ by this plane is the same as the section of each $W_{2}$ of the pencil. But each $W_{2}$ contains the 15 lines joining its hexad of nodes. Hence the $\infty^{1}$ sextics $\lambda(\omega t)^{6}+\left[(\mu t)^{3}\right]^{2}$ determine on $N^{3} \infty^{1}$ sixpoints whose complete figures are cut by a plane in the quartic curve $Q$ containing $\infty^{1}$ configurations $\left(15_{3}, 20_{4}\right)$.

This notion of a pencil of $W_{2}$ 's cannot be extended. The method which I am about to give not only yields additional information with respect to the section of $W_{2}$ but also applies to the section of $W_{p}$ in $S_{2 p-1}$ by a $(p+1)$-secant $S_{p}$ of $N_{2 p-1}$. Taking $(\omega t)^{6}=f_{3}{ }^{2}-f_{2} f_{4}$, we have already indicated the roots $t_{1}, \cdots, t_{6}$ of $(\omega t)^{6}$, and the roots $r_{1}, r_{2}$ of $f_{2}$. Let also $s_{1}, s_{2}, s_{3}$ be the roots of $f_{3}$, so that

$$
f_{3}=c\left(t s_{1}\right)\left(t s_{2}\right)\left(t s_{3}\right) .
$$

For variation of the constant factor $c$ in $f_{3}$ we have in

$$
(\omega t)^{6}-f_{3}^{2}=-f_{2} f_{4}
$$

a pencil of binary sextics and $f_{2}$ is a duad in some hexad of the pencil. The seventh powers of the 9 linear forms, $\left(t t_{i}\right),\left(t s_{j}\right)$, are connected by a linear identity, and the polar of $f_{4}$ as to this identity yields the following identity: 


$$
\sum_{i=1}^{i=6} \frac{\left(t t_{i}\right)^{3} \cdot f_{4}\left(t_{i}\right)}{f_{3}\left(t_{i}\right) \cdot \omega^{\prime}\left(t_{i}\right)}+\sum_{j=1}^{j=3} \frac{\left(t s_{j}\right)^{3} \cdot f_{4}\left(s_{j}\right)}{\omega\left(s_{j}\right) \cdot f_{3}^{\prime}\left(s_{j}\right)} \equiv 0 .
$$

Since $f_{4}\left(t_{i}\right) / f_{3}\left(t_{i}\right)=f_{3}\left(t_{i}\right) / f_{2}\left(t_{i}\right)$, we see by comparison with (A) that

$$
-(\alpha t)^{3}=\sum_{j=1}^{j=3} \frac{\left(t s_{j}\right)^{3} \cdot f_{4}\left(s_{j}\right)}{\omega\left(s_{j}\right) \cdot f_{3}^{\prime}\left(s_{j}\right)}
$$

Since also $f_{4}\left(s_{j}\right) / \omega\left(s_{j}\right)=-1 / f_{2}\left(s_{j}\right)$,

$$
(\alpha t)^{3}=\sum_{j=1}^{j=3} \frac{\left(t s_{j}\right)^{3}}{f_{2}\left(s_{j}\right) \cdot f_{3}^{\prime}\left(s_{j}\right)} .
$$

Hence, for given trisecant plane $\pi=s_{1} s_{2} s_{3}$ of $N^{3}$, and for a selected duad $f_{2}$, in some hexad of the pencil above, there is a point $(\alpha t)^{3}$ on $W_{2}$ which, according to (C), is on the plane $\pi$, and, according to (B), is on the bisecant $r_{1} r_{2}$ of $N^{3}$ determined by $f_{2}$. This proves the Morley-Conner theorem that the section $Q$ of $W_{2}$ by $\pi$ contains the inscribed configurations which they observed.

But the three-term expression (D) contributes new information. It contains the variable duad $f_{2}$ of the pencil in the denominator. Hence the point of $Q$ on the left is the quadratic transform of the point $f_{2}(t)$ in a plane $\pi^{\prime}$. This yields the following theorem.

If the hexads of the pencil, $(\omega t)^{6}-f_{3}^{2}=0$, be plotted as hexads of tangents of a norm-conic $N^{2}$ in a plane $\pi^{\prime}$, the locus of the vertices of the complete hexagons is a quintic curve with three nodes whose joins are the three tangents $f_{3}=0$ of $N^{2}$; and the transform of this quintic by a quadratic transformation with $F$-points at the nodes is a section $Q$ of $W_{2}$ by the plane $f_{3}=0$ with the configurations indicated.

These results can be generalized immediately to apply to the curves in which $W_{p}$ is cut by the $(p+1)$-secant $S_{p}$ 's of $N^{2 p-1}$ determined by $f_{p+1}$. They can also be extended to cover the cases where the $(p+1)$-secant $S_{p}$ passes through some of the points of $P_{2 p+2}^{2 p-1}$. Suppose, for example, that $j+2$ of the branch points, determined by $\lambda_{j+2}(t)=0$, are on the line $y_{0}=0$. Then $\lambda_{j+2}$ is a factor of $f_{p+2}$, of $(\omega t)^{2 p+2}$, and therefore of $f_{p+1}$. If we set

$$
(\omega t)^{2 p+2}=\mu_{2 p-j} \cdot \lambda_{j+2}, f_{p+2}=g_{p-j} \cdot \lambda_{j+2}, f_{p+1}=g_{p-j-1} \cdot \lambda_{j+2},
$$


the pencil, analogous to the above pencil of sextics, becomes

$$
\mu_{2 p-j}-g_{p-j-1}^{2} \cdot \lambda_{j+2}=-f_{p} \cdot g_{p-j} .
$$

For $j=-2$ we have the generic case mentioned above. For $j=-1, j=0$ we have sections of $W_{p}$ by $S_{p}$ 's on one or two points of $P_{2 p+2}^{2 p-1}$. For $j=1,2, \cdots, p$ we have curves on $W_{p}$ which also lie on the $F$-spaces of the first, second, $\cdots, p$ th kind.

I may say finally that the importance of $W_{p}$ lies in the information which it furnishes with respect to those properties which differentiate the hyperelliptic $K_{p}$ from the $K_{p}$ attached to more general theta functions.

The study of this manifold has had for me a peculiar fascination because of the demand it makes on geometric ingenuity. General methods of attack have been quite uniformly fruitless because of one or another special characteristic of the manifold. Doubtless we all are accustomed to seeing the waste basket claim the major part of our labour. In this respect at least I may justly assert that $W_{p}$ is the most outstanding topic I have ever studied.

UNIVERSITY OF ILLINOIS 